SUNY-NTG-95-55

\title{
Nucleons at Finite Temperature
}

\author{
M. Kacir and I. Zahed \\ Department of Physics \\ State University of New York at Stony Brook \\ Stony Brook, New York 11794-3800
}

\begin{abstract}
The nucleon mass shift is calculated using chiral counting arguments and a virial expansion, without and with the $\Delta$. At all temperatures, the mass shift and damping rate are dominated by the $\Delta$. Our results are compared with the empirical analysis of Leutwyler and Smilga, as well as results from heavy baryon chiral perturbation theory in the large $N_{c}$ (number of color) limit. We show that unitarity implies that the concepts of thermal shifts are process dependent.
\end{abstract}

SUNY-NTG-95-55

December 1995 


\section{Introduction}

In dilute many-body systems, particles undergoing multiple rescattering behave as quasiparticles. As a result, their mass and width depart from the vacuum values. While varying widths are a direct measurement of quasiparticle absorption, and may be important for transport properties, varying mass shifts may have fundamental consequences on threshold behaviours, and particle spectra [1].

Non-perturbative model calculations at finite temperature and/or density support the idea that hadronic masses and widths change as a function of temperature and density [2]. The idea that a changing rho mass in the hadronic medium [3], may be key in understanding the excess of dileptons (CERES [4]) and dimuons (HELIOS [5]) below the rho mass in present relativistic heavy-ion collisions.

The definition of quasiparticle masses is, however, process dependent. At finite temperature a quasiparticle mass can be defined by using the free energy (energy mass), space-like correlation functions (screening mass), or time-like correlation functions (pole mass), to cite a few.

The purpose of this letter is to investigate the effects of temperature on nucleons in a heat bath of pions. In section 2, we exploit a virial type expression for the mass shift and the damping rate in terms of the forward scattering amplitude. This amplitude is obtained following arguments based on chiral power counting arguments and on-shell Ward identities [6]. In section 3, we give a full account of the role played by the $\Delta$ resonance. A comparison with results based on empirical data is achieved [7]. In section 4, we discuss the large $N_{c}$ limit of our results, then compare with recent results from heavy baryon chiral perturbation theory $(\mathrm{HB} \chi \mathrm{PT})$ [8]. In section 5, using a variant of the Bethe-Uhlenbeck argument, we show that for the nucleon its mass shift is distinct from its energy shift. In general, unitarity implies that the concepts of thermal shifts are process dependent. In section 6, we summarize our conclusions.

\section{Ward-Identity}

A nucleon immersed in a heat bath of pions undergoes rescattering and absorption. For temperatures $T \sim m_{\pi}$, the pion mean free path is about $2-3 \mathrm{fm}$. As a result the 
pion thermal density is about $(1 / 3)^{3} \mathrm{fm}^{-3}$. The pion gas is dilute. Hence, pion-nucleon collisions in the heat bath may be organized in terms of the pion density (virial expansion). To leading order in the pion density, the nucleon pole mass receives a coherent shift $\Delta m_{N}(p)$ and damping rate $\gamma_{N}^{T}(p)$ given by

$$
\Delta m_{N}(p)-\frac{i}{2} \gamma_{N}^{T}(p)=-\sum_{a=1}^{3} \int \frac{d^{3} k}{(2 \pi)^{3}} \frac{1}{e^{\omega_{k} / T}-1} \frac{\mathcal{T}^{a a}(p, k)}{2 \omega_{k}}
$$

where $\mathcal{T}^{a a}$ is the forward pion-nucleon scattering amplitude, and $\omega_{k}=\sqrt{k^{2}+m_{\pi}^{2}}$. In throughout this work, we respectively denote the pion momentum by $k$ and $q$ in the laboratory and center of mass frame. $\mathcal{T}^{a a}$ is available from pion-nucleon scattering data [9], and was used by Leutwyler and Smilga to assess the shift in the nucleon mass at zero momentum [7]. Here, we will instead rely on on-shell Ward identities and chiral power counting to derive an expression for (四) at tree level. This will help us assess the various thermal contributions to the mass and width of the nucleon, as well as the importance of the $\Delta$.

We note that the pion-nucleon scattering amplitude $\mathcal{T}$ can be reduced using Weinberg's formula [10]. Taking $(k, a)$ as the incoming pion and $(k, b)$ the outgoing pion, the amplitude, associated to the scattering process of Fig. 1, reads

$$
\mathcal{T}=\mathcal{T}_{V}+\mathcal{T}_{S}+\mathcal{T}_{A A}
$$

where

$$
\begin{aligned}
\mathcal{T}_{V}^{a b} & =+\frac{i}{f_{\pi}^{2}} k^{\mu} \epsilon^{b a c}\left\langle N(p)\left|\mathbf{V}_{\mu}^{c}(0)\right| N(p)\right\rangle \\
\mathcal{T}_{S}^{a b} & =-\frac{m_{\pi}^{2}}{f_{\pi}} \delta^{a b}\langle N(p)|\sigma(0)| N(p)\rangle_{\text {conn. }}=+\frac{\sigma_{\pi N}}{f_{\pi}^{2}} \delta^{a b} \\
\mathcal{T}_{A A}^{a b} & =+\frac{i}{f_{\pi}^{2}} k^{\mu} k^{\nu} \int d^{4} x e^{-i k \cdot x}\left\langle N(p)\left|T^{*} \mathbf{j}_{A \mu}^{a}(x) \mathbf{j}_{A \nu}^{b}(0)\right| N(p)\right\rangle_{\text {conn. }} .
\end{aligned}
$$

Here $\mathbf{V}$ is the quantum vector current, $\sigma$ the quantum scalar density, and $\mathbf{j}_{A \mu}^{a}$ the quantum one-pion reduced axial-current. Their form can be found in Refs. [6, 11] for QCD and sigma models. In the forward amplitude (2), the isovector charge of the nucleon (3) drops, and one is left with the sigma-term contribution (田) and the one-pion reduced axial-axial correlator (5). Thus

$$
\sum_{a=1}^{3} \mathcal{T}^{a a}=3 \frac{\sigma_{\pi N}}{f_{\pi}^{2}}+\frac{i}{f_{\pi}^{2}} k^{\mu} k^{\nu} \int d^{4} x e^{-i k \cdot x} \sum_{a=1}^{3}\left\langle N(p)\left|T^{*} \mathbf{j}_{A \mu}^{a}(x) \mathbf{j}_{A \nu}^{a}(0)\right| N(p)\right\rangle_{\text {conn }}
$$


Inserting (6) into (1) we obtain for the mass shift and damping rate

$$
\begin{aligned}
\Delta m_{N}(p)-\frac{i}{2} \gamma_{N}^{T}(p)= & -3 n_{\pi}(T) \frac{\sigma_{\pi N}}{f_{\pi}^{2}}-\sum_{a=1}^{3} \int \frac{d^{3} k}{(2 \pi)^{3}} \frac{1}{2 \omega_{k}} \frac{1}{e^{\omega_{k} / T}-1} \\
& \times \frac{i}{f_{\pi}^{2}} k^{\mu} k^{\nu} \int d^{4} x e^{-i k \cdot x}\left\langle N(p)\left|T^{*} \mathbf{j}_{A \mu}^{a}(x) \mathbf{j}_{A \nu}^{a}(0)\right| N(p)\right\rangle_{\text {conn. }}
\end{aligned}
$$

where

$$
3 n_{\pi}(T)=\sum_{a=1}^{3} \int \frac{d^{3} k}{(2 \pi)^{3}} \frac{1}{2 \omega_{k}} \frac{1}{e^{\omega_{k} / T}-1}=\sum_{a=1}^{3} \int \frac{d^{4} k}{(2 \pi)^{4}} 2 \pi \delta\left(k^{2}-m_{\pi}^{2}\right) \frac{1}{e^{\left|k^{0}\right| / T}-1}
$$

counts the number of pions per unit energy and per unit volume in the heat bath. For massless pions, this is just $T^{2} / 4$. To assess the role of the one-pion reduced axial-axial correlator, we will use power counting in $1 / f_{\pi}$ as discussed in Refs. [6, 11] with and without the $\Delta$.

In the absence of $\Delta$, the one-pion reduced axial-current at tree level reads [11]

$$
\mathbf{j}_{A \mu}^{a}=g_{A} \overline{\mathbf{\Psi}} \gamma_{\mu} \gamma_{5} \frac{\tau^{a}}{2} \boldsymbol{\Psi}+\frac{\sigma_{\pi N}}{m_{\pi}^{2}} \partial_{\mu}\left(\overline{\mathbf{\Psi}} i \gamma_{5} \tau^{a} \boldsymbol{\Psi}\right)+\mathcal{O}\left(\frac{1}{f_{\pi}^{2}}\right)
$$

where $g_{A}=1.357$ is the nucleon axial-coupling and $\boldsymbol{\Psi}$ is the nucleon-field. We take $m_{\pi}=139$ $\mathrm{MeV}$ and $m_{N}=939 \mathrm{MeV}$ for the pion and nucleon mass, the empirical value $\sigma_{\pi N}=45 \pm 8$ $\mathrm{MeV}$ and the pion decay constant $f_{\pi}=94 \mathrm{MeV}$. Our expansion is on-shell, so all the parameters in our expressions are set at their physical (on mass-shell renormalization). The corrections follow from pion and nucleon loops [11]. Inserting (9) into the scattering amplitude (5) yields

$$
\begin{aligned}
\mathcal{T}_{N}(\nu) & =\sum_{a=1}^{3} \mathcal{T}_{A A}^{a a} \\
& =-\frac{3}{4 f_{\pi}^{2}}\left(4 g_{A}\left(2 \sigma_{\pi N}-g_{A} m_{N}\right)-\frac{\left(\sigma_{\pi N}-g_{A} m_{N}\right)^{2}}{2 m_{N}}\left(\frac{4 \nu}{\nu-\nu_{N}}+\frac{4 \nu}{\nu+\nu_{N}}\right)\right) \\
& +\mathcal{O}\left(\frac{1}{f_{\pi}^{4}}\right)
\end{aligned}
$$

where $\nu=p \cdot k / m_{N}=\left(E_{q} \omega_{q}+q^{2}\right) / m_{N}$ and $\nu_{N}=m_{\pi}^{2} / 2 m_{N}$. If we note that to this order the pion-nucleon $\mathrm{S}$-wave scattering length is given by

$$
4 \pi\left(1+\frac{m_{\pi}}{m_{N}}\right) a^{+}=\frac{\sigma_{\pi N}}{f_{\pi}^{2}}+\frac{1}{3} \mathcal{T}_{N}\left(m_{\pi}\right)
$$


then the nucleon mass shift and damping rate (7) read to order $\mathcal{O}\left(1 / f_{\pi}^{2}\right)$, in terms of real and imaginary parts of the scattering amplitude

$$
\begin{aligned}
\Delta m_{N}(0)= & -3 n_{\pi}(T)\left(1+\frac{m_{\pi}}{m_{N}}\right) 4 \pi a^{+} \\
& -\int \frac{d^{3} k}{\left(2 \pi^{3}\right)} \frac{1}{2 \omega_{k}} \frac{1}{e^{\omega_{k} / T}-1} \operatorname{Re}\left(\mathcal{T}_{N}\left(\omega_{k}\right)-\mathcal{T}_{N}\left(m_{\pi}\right)\right)+\mathcal{O}\left(\frac{1}{f_{\pi}^{4}}\right)
\end{aligned}
$$

and

$$
\gamma_{N}^{T}(0)=2 \int \frac{d^{3} k}{\left(2 \pi^{3}\right)} \frac{1}{2 \omega_{k}} \frac{1}{e^{\omega_{k} / T}-1} \operatorname{Im}\left(\mathcal{T}_{N}\left(\omega_{k}\right)-\mathcal{T}_{N}\left(m_{\pi}\right)\right)+\mathcal{O}\left(\frac{1}{f_{\pi}^{4}}\right)
$$

This is an exact result of chiral symmetry. At tree level, and in the absence of the isobar, there is no absorption and the damping rate vanishes. Each contribution from both the scattering length term and the nucleon is displayed in Fig. 3. For temperatures $T<m_{\pi}$, they are opposite in sign and of the order of $1 \mathrm{MeV}$ resulting in a nucleon mass shift which is negative and less than $1 \mathrm{MeV}$ over this range of temperature.

\section{$3 \Delta$ resonance}

The role of the $\Delta$ with a finite width cannot be assessed uniquely in the formalism developed in Refs. [6, 11]. Given its importance in the axial-axial correlator, we will try to approximately assess its contribution in this section. We will assume that the $\Delta$ has a zero width (exact argument) and then assign by hand a width in the final result (approximate argument). With this in mind, the pion-nucleon-delta coupling will be taken to be pseudovector with strength,

$$
\frac{g_{\pi N \Delta}}{2 m_{\Delta}}\left(\partial_{\mu} \pi^{a}\right)\left(\bar{\Psi} \mathrm{Q}^{\mu, a}+\text { h.c. }\right)
$$

where $\pi^{a}$ is the quantum PCAC pion field [6, 11], and $\mathrm{Q}^{\mu, a}$ is a Rarita-Schwinger field 12 with vector index $\mu$ and isospin index $a$. The (omitted) Dirac and isodoublet indices are contracted over with the nucleon field. The transition matrix element between the nucleon with momentum $p$ and isospin $b$, and the isobar with momentum $p^{\prime}$ and isospin $b^{\prime}$, induced by the one-pion reduced axial-vector current takes the general form

$$
\begin{aligned}
\left\langle N(p, b)\left|\mathbf{j}_{A \mu}^{a}(0)\right| \Delta\left(p^{\prime}, b^{\prime}\right)\right\rangle= & \bar{u}(p, b)\left(F\left(k^{2}\right) g_{\mu \nu}+G\left(k^{2}\right) \gamma_{\mu} k_{\nu}\right. \\
& \left.+H\left(k^{2}\right) k_{\mu} k_{\nu}+i I\left(k^{2}\right) \sigma_{\mu}^{\lambda} k_{\lambda} k_{\nu}\right) \mathbf{U}^{\nu, a}\left(p^{\prime}, b^{\prime}\right)
\end{aligned}
$$


where $k^{2}=\left(p-p^{\prime}\right)^{2}$ and $\mathbf{U}$ is a Rarita-Schwinger spinor. The general structure (15) follows from Lorentz invariance, P- and T-invariance [13]. At tree level, the form factors obey a Goldberger-Treiman relation

$$
\frac{g_{\pi N \Delta}}{2 m_{\Delta}}=\frac{F+G\left(m_{\Delta}-m_{N}\right)}{f_{\pi}}
$$

where $F=F(0)$ and $G=G(0)$. The contribution of the $\Delta$ to the one-pion reduced axial-axial correlator in the nucleon (7) can be evaluated using (15) and the following $\Delta$ propagator

$$
\begin{aligned}
\boldsymbol{\Delta}_{\mu \nu}^{a b}(p)= & \left(\delta^{a b}-\frac{1}{3} \tau^{a} \tau^{b}\right) \frac{1}{\not p-m_{\Delta}} \\
\times & \left(-g_{\mu \nu}+\frac{1}{3} \gamma_{\mu} \gamma_{\nu}-\frac{2}{3 m_{\Delta}^{2}} p_{\mu} p_{\nu}+\frac{1}{3 m_{\Delta}}\left(\gamma_{\mu} p_{\nu}-p_{\mu} \gamma_{\nu}\right)\right)
\end{aligned}
$$

In Fig. 2, we exhibit the scattering process considered in the heat bath. The resulting amplitude is

$$
\begin{aligned}
\mathcal{T}_{\Delta}(\nu)= & -\frac{4}{3 f_{\pi}^{2}} \Lambda(\nu)\left(-G^{2}\left(\nu+m_{\Delta}-m_{N}\right)+\frac{g_{\Delta}^{2}}{2 m_{N}} \frac{\nu+m_{\Delta}+m_{N}}{\nu-\nu_{\Delta}}\right) \\
& +(\nu \rightarrow-\nu)+\mathcal{O}\left(\frac{1}{f_{\pi}^{4}}\right)
\end{aligned}
$$

where $\nu_{\Delta}=\left(m_{\Delta}^{2}-m_{N}^{2}-m_{\pi}^{2}\right) / 2 m_{N}, g_{\Delta}^{2}=\left(F^{2}-G^{2}\left(m_{\Delta}-m_{N}\right)^{2}\right)$ and

$$
\Lambda(\nu)=m_{\pi}^{2}-\frac{\left(m_{N} \nu+m_{\pi}^{2}\right)^{2}}{m_{\Delta}^{2}}
$$

This is to be compared with the nucleon contribution (10) discussed above.

In terms of (10) and (18) the contribution to order $\mathcal{O}\left(1 / f_{\pi}^{4}\right)$ to the isospin averaged pion-nucleon scattering amplitude (6) reads

$$
\sum_{a=1}^{3} \mathcal{T}^{a a}(\nu)=12 \pi a^{+}\left(1+\frac{m_{\pi}}{m_{N}}\right)+\left(\mathcal{T}_{N}(\nu)+\mathcal{T}_{\Delta}(\nu)-\mathcal{T}_{N}\left(m_{\pi}\right)-\mathcal{T}_{\Delta}\left(m_{\pi}\right)\right)
$$

where the S-wave scattering length now receives a contribution from the isobar through

$$
4 \pi a^{+}\left(1+\frac{m_{\pi}}{m_{N}}\right)=\frac{\sigma_{\pi N}}{f_{\pi}^{2}}+\frac{1}{3} \mathcal{T}_{N}\left(m_{\pi}\right)+\frac{1}{3} \mathcal{T}_{\Delta}\left(m_{\pi}\right)
$$


In terms of the real and imaginary parts of the scattering amplitude in (20), the nucleon mass shift is given by ( $p=0$ is understood)

$$
\begin{aligned}
\Delta m_{N}= & -3 n_{\pi}(T)\left(1+\frac{m_{\pi}}{m_{N}}\right) 4 \pi a^{+} \\
& -\int \frac{d^{3} k}{\left(2 \pi^{3}\right)} \frac{1}{2 \omega_{k}} \frac{1}{e^{\omega_{k} / T}-1} \operatorname{Re}\left(\mathcal{T}_{N}\left(\omega_{k}\right)+\mathcal{T}_{\Delta}\left(\omega_{k}\right)-\mathcal{T}_{N}\left(m_{\pi}\right)-\mathcal{T}_{\Delta}\left(m_{\pi}\right)\right) \\
& +\mathcal{O}\left(\frac{1}{f_{\pi}^{4}}\right)
\end{aligned}
$$

and the damping rate by

$$
\begin{aligned}
\gamma_{N}^{T}= & +2 \int \frac{d^{3} k}{\left(2 \pi^{3}\right)} \frac{1}{2 \omega_{k}} \frac{1}{e^{\omega_{k} / T}-1} \operatorname{Im}\left(\mathcal{T}_{N}\left(\omega_{k}\right)+\mathcal{T}_{\Delta}\left(\omega_{k}\right)-\mathcal{T}_{N}\left(m_{\pi}\right)-\mathcal{T}_{\Delta}\left(m_{\pi}\right)\right) \\
& +\mathcal{O}\left(\frac{1}{f_{\pi}^{4}}\right)
\end{aligned}
$$

This result relies on the choice of the interaction (14), and as such is not unique. Since the $\Delta$ sits in the continuum, (22) diverges. In nature, $\Delta$ has a width $\Gamma_{\Delta}$ and (22) is finite. To account for this width, we parameterize the resonant part of the scattering amplitude (17) in the P33 channel by a Breit-Wigner form.

To this end, we decompose the amplitude (18) as follows 14

$$
\mathcal{T}_{\Delta}(\nu)=3\left(A_{\Delta, p}^{+}+\nu B_{\Delta, p}^{+}+A_{\Delta, n p}^{+}+\nu B_{\Delta, n p}^{+}\right)
$$

where the pole parts

$$
\begin{aligned}
& A_{\Delta, p}^{+}(\nu)=A_{\Delta, d p}^{+}(\nu)+A_{\Delta, c p}^{+}(\nu)=\frac{g_{\Delta}^{2}}{9 m_{N}} \alpha_{1}\left(\frac{1}{\nu_{\Delta}-\nu}+\frac{1}{\nu_{\Delta}+\nu}\right) \\
& B_{\Delta, p}^{+}(\nu)=B_{\Delta, d p}^{+}(\nu)+B_{\Delta, c p}^{+}(\nu)=\frac{g_{\Delta}^{2}}{9 m_{N}} \beta_{1}\left(\frac{1}{\nu_{\Delta}-\nu}-\frac{1}{\nu_{\Delta}+\nu}\right)
\end{aligned}
$$

and the non-pole parts

$$
\begin{aligned}
A_{\Delta, n p}^{+}(\nu)= & -\frac{4 g_{\Delta}^{2}}{9 m_{\Delta}}\left(\left(E_{\Delta}+m_{N}\right)\left(2 m_{\Delta}-m_{N}\right)+m_{\pi}^{2}\left(2+m_{N} / m_{\Delta}\right)\right) \\
+ & \frac{4 G^{2}}{9 f_{\pi}^{2}} 2\left(m_{\Delta}-m_{N}\right)\left(m_{\pi}^{2}+\frac{m_{N}^{2} \nu^{2}+m_{\pi}^{4}}{m_{\Delta}^{2}}\right) \\
& B_{\Delta, n p}^{+}(\nu)=-\frac{4 g_{\Delta}^{2}}{9 m_{\Delta}} \frac{m_{N} \nu}{m_{\Delta}}+\frac{16 G^{2}}{9 f_{\pi}^{2}} \frac{m_{N} \nu m_{\pi}^{2}}{m_{\Delta}^{2}}
\end{aligned}
$$


with

$$
\begin{aligned}
g_{\Delta}^{2} & =\left(F^{2}-G^{2}\left(m_{\Delta}-m_{N}\right)^{2}\right) / f_{\pi}^{2} \\
\alpha_{1} & =3\left(m_{N}+m_{\Delta}\right)\left(E_{\Delta}^{2}-m_{N}^{2}\right)+\left(m_{\Delta}-m_{N}\right)\left(E_{\Delta}+m_{N}\right)^{2} \\
\beta_{1} & =3\left(E_{\Delta}^{2}-m_{N}^{2}\right)-\left(E_{\Delta}+m_{N}\right)^{2} \\
E_{\Delta} \pm m_{N} & =\frac{1}{2 m_{\Delta}}\left(\left(m_{\Delta} \pm m_{N}\right)^{2}-m_{\pi}^{2}\right)
\end{aligned}
$$

The procedure for introducing the width of the $\Delta$ follows the approach in [15] and is discussed in details in the appendix.

Using the empirical values $\sigma_{\pi N}=45 \pm 8 \mathrm{MeV}$ [16], $a^{+}=-(8 \pm 4) 10^{-3} / m_{\pi}$ [9], and the $\mathrm{SU}(3)$ relation $g_{\pi N \Delta}=3 g_{\pi N N} / \sqrt{2}$, the Goldberger-Treiman relation (16) and the expression (21) of the scattering length $a^{+}$in terms of threshold amplitudes allow for a determination of the isobar axial-form factors $F$ and $G$. We have examined the resulting amplitude opposite to the experimental data [14] and noticed a very poor fit at low energy. Instead, we rely on the empirical values at threshold of $A^{+}(q=0)=227.3 \mathrm{GeV}^{-1}$ and $B^{+}(q=0)=-1639 \mathrm{GeV}^{-1}$. The results are $F=1.382$ and $G=4.23510^{-4} \mathrm{MeV}^{-1}$. The first is to be compared with $F=3 g_{A} / 2 \sqrt{2} \sim 1.31$ from large $N_{c}$ arguments [13]. In Figs. 4 and 5, we display both real and imaginary parts of $A^{+}(\nu)$ and $B^{+}(\nu)$ opposite the empirical data from [14].

The mass shift of the $\Delta$ follows from (22) after inserting (20) and re-expressing the phase space integral from the nucleon rest frame, to the center of mass frame. The various contributions to the nucleon mass shift, stemming from the scattering length, the nucleon Born term, the $\Delta$ Born term (with width), and the combined sum, are shown in Fig. 3. In Fig. 6, the total mass shift $\Delta m_{N}$ (full line) is compared to the one (dotted line) obtained from the empirical scattering amplitude [14]. The rise in the nucleon mass at temperatures of the order of the pion mass is conditioned by the resonance. The width of the $\Delta$ yields an imaginary part to the mass shift. Nucleons in the heat bath undergo strong absorption through $\pi N \rightarrow \Delta$. In Fig. 7, we display the damping rate $\gamma_{N}^{T}$ (full line) opposite to the empirical one (dotted line). We point out that the nucleon mass shift was obtained in the same manner by Leutwyler and Smilga [7]. For the damping rate, use of the total $\pi p$ cross section was made in [7]. Overall, we can observe that our chiral counting argument for the scattering amplitude allows us to reproduce quantitatively both the nucleon mass 
shift and damping rate up to temperatures of the order of $\mathrm{m}_{\pi}$. As already pointed out in [7] for higher temperatures, we would expect other contributions like the $\mathrm{N}^{*}$ to play a role. In this regime, however, the pion gas is no longer dilute and the use of the first two terms in the virial expansion is no longer justified.

\section{Large $N_{c}$ limit}

In this section, we will examine the forward scattering amplitude in the large $N_{c}$ limit. As we will see below, the contribution of the width being subleading in $N_{c}$, we will ignore it in the discussion for the mass shift $\Delta m_{N}$. With $m_{N}, m_{\Delta}, \sigma_{\pi N}, g_{A}, f_{\pi}^{2}, F$ and $G$ all of order $\mathcal{O}\left(N_{c}\right)$ and denoting $\mu=m_{\Delta}-m_{N} \sim \mathcal{O}\left(1 / N_{c}\right)$, we can write for the forward scattering amplitude for the nucleon

$$
\mathcal{T}_{N}(\nu) \sim \frac{3 g_{A}^{2} m_{N}}{f_{\pi}^{2}} \frac{\nu_{N}^{2}}{\nu^{2}-\nu_{N}^{2}} \sim \mathcal{O}\left(N_{c}^{0}\right)
$$

and for the $\Delta$

$$
\mathcal{T}_{\Delta}(\nu) \sim \frac{4}{3 f_{\pi}^{2}} \nu^{2}\left(-2 G^{2} \mu+\frac{F^{2}}{m_{N}}+\frac{F^{2}}{m_{N}} \frac{2 m_{N} \mu}{\nu^{2}-\mu^{2}}\right) \sim \mathcal{O}\left(N_{c}^{0}\right)
$$

The contribution from the sigma term is

$$
\mathcal{T}_{\sigma}(\nu) \sim \frac{3 \sigma_{\pi N}}{f_{\pi}^{2}} \sim \mathcal{O}\left(N_{c}^{0}\right)
$$

Setting $m_{\pi}=0$, we express the mass shift $\Delta m_{N}=\Delta m_{N, n p}+\Delta m_{N, p}$ where the subscripts $n p$ and $p$ respectively indicate non-pole and pole contributions. The non-pole part reads

円

$$
\Delta m_{N, n p}=-\frac{T^{2}}{4 \pi^{2} f_{\pi}^{2}} \int_{0}^{\infty} \frac{x d x}{e^{x}-1}\left(3 \sigma_{\pi N}-\frac{4}{3} x^{2} T^{2}\left(-2 G^{2} \mu+\frac{F^{2}}{m_{N}}\right)\right)
$$

The pole part reads

$$
\Delta m_{N, p}=\frac{2 F^{2}}{3} \frac{\mu T^{2}}{\pi^{2} f_{\pi}^{2}} \int_{0}^{\infty} d x \frac{x^{3}}{x^{2}-\mu^{2} / T^{2}} \frac{1}{e^{x}-1}
$$

Both pole and non-pole contributions are of order $\mathcal{O}\left(N_{c}^{0}\right)$. The mass shift obtained can be compared to the result quoted in [8], where heavy baryon chiral perturbation theory $(\mathrm{HB} \chi \mathrm{PT})$ arguments were used. The non-pole term is absent in [8] and the result quoted

\footnotetext{
${ }^{1}$ Note that the term $\mu / T$ in $\Delta m_{N, p}$, is subleading in $1 / N_{c}$ and should be effectively dropped. We have kept it to make the comparison with [8] immediate.
} 
for the pole term has $2 F^{2} / 3$ substituted by $\chi_{N} g^{2}$ where $\chi_{N}=15$ and $g=3(F+D) / 5$. This mismatch in the factor $2 F^{2} / 3$ and the absence of a non-pole term may explain a mass shift with essentially the same trend as ours, however from $\left[\mathbb{8}, \Delta m_{N}\right.$ is larger than zero for $T>80 \mathrm{MeV}$. In Fig. 8, we display the pole and non-pole contributions to the mass shift. The short-dashed line represents the sum of the pole and non-pole contribution and the long-dashed line the mass shift from chiral power counting of Fig. 6.

In order to examine the damping rate in the large $N_{c}$ limit, we first write the P33 wave for the direct term in the forward scattering amplitude (see appendix)

$$
f_{33}^{P} \sim \frac{F^{2}}{12 \pi f_{\pi}^{2}} \frac{q^{2}}{\nu_{\Delta}-\nu}
$$

Using a Breit-Wigner form to describe the width of the $\Delta$ and keeping in mind that this is valid at resonance, the imaginary part of the P33 wave reads

$$
\operatorname{Im} f_{33}^{P}=\frac{\Gamma_{\Delta}}{2 q} \frac{\Gamma_{\Delta} / 2}{\left(\nu_{\Delta}-\nu\right)^{2}+\left(\Gamma_{\Delta} / 2\right)^{2}}
$$

where, in the large $N_{c}$ limit, the width reads

$$
\Gamma_{\Delta}=\frac{F^{2}}{6 \pi f_{\pi}^{2}} \mu^{3} \sim \mathcal{O}\left(1 / N_{c}^{2}\right)
$$

Due to the order of $\Gamma_{\Delta}$ in (37), we can further write the P33 wave

$$
\operatorname{Im} f_{33}^{P}=\frac{\Gamma_{\Delta}}{2 q} \pi \delta(q-\mu)
$$

It is remarkable that the leading term in $N_{c}$ for $\operatorname{Im} f_{33}^{P}$ is exactly the result obtained from the pole approximation approach to the width of the $\Delta$ as a narrow resonance [17]. With this in hand, it is not difficult to obtain the leading term in $N_{c}$ for the imaginary part of the forward scattering amplitude. The result is

$$
\operatorname{Im} \mathcal{T}_{\Delta}(k)=\frac{4 \pi}{3} \frac{F^{2}}{f_{\pi}^{2}} \mu^{2} \delta(k-\mu)
$$

The damping rate follows from (23) and is of order $\mathcal{O}\left(1 / N_{c}^{2}\right)$

$$
\gamma_{N}^{T}=\frac{2 F^{2}}{3} \frac{\mu^{3}}{\pi f_{\pi}^{2}} \frac{1}{e^{\mu / T}-1}
$$

Again, if we attempt a comparison with [8], we need to substitute $2 F^{2} / 3$ by $\chi_{N} g^{2} / 12$. In Fig. 9, we display the damping rate (40) (full line) opposite the result obtained from chiral power counting (long-dashed line).

The present large $N_{c}$ results for the nucleon mass shift and damping rate, can be effectively tested in the Skyrme model. This point will be discussed elsewhere 18. 


\section{Process Dependence}

The above definition of the mass shift relies on the pole mass definition. Is it definition (process) independent ? In this section we will show that in general unitarity forces the concept of thermal shifts to be process dependent, weakly when "perturbative" cuts are involved and strongly when "resonant" cuts are involved. Our argument is generic, although we will use the nucleon for illustration.

Let $\Delta E_{N}$, be the shift in the energy of a single nucleon immersed in a dilute heat bath of pions. To first order in the pion density, the shift is

$$
\Delta E_{N}=\int_{0}^{+\infty} \frac{d k}{\pi} \sum_{I, l}(2 l+1) \delta_{I, l}^{\prime}(k) \frac{\omega_{k}}{e^{\omega_{k} / T}-1}
$$

where $\delta_{l, I}$ is the phase-shift of a pion partial wave carrying angular momentum $l$ and isospin $I$, expressed in the rest frame of the nucleon. (41) is just the thermal zero-point energy if we recall that in a spherical box of size $R$, the boundary condition on the lth partial wave is

$$
k R-l \frac{\pi}{2}+\delta_{I, l}(k)=n \pi
$$

where $n$ is integer. Hence, the change in the number of states per unit $k$ is $d n / d k=$ $\delta_{I, l}^{\prime}(k) / \pi$. Hence (41). In terms of the partial phase shifts $\delta_{I, l}$, the scattering amplitude $\mathcal{T}_{I}(k)$ for fixed isospin $I$ (our $\mathcal{T}^{a a}$ above), is given by the conventional spherical expansion

$$
\mathcal{T}_{I}(k)=i \frac{2 \pi}{k} \sum_{l}(2 l+1)\left(e^{2 i \delta_{I, l}(k)}-1\right) P_{l}(\hat{k})
$$

Using (43), we can rewrite (41) in terms of the scattering amplitude, a procedure known from the work of Bethe and Uhlenbeck [19]. The result is

$$
\begin{aligned}
\Delta E_{N}=+ & \sum_{I} \int \frac{d^{3} k}{(2 \pi)^{3}} \frac{\operatorname{Re} \mathcal{T}(k)}{2 \omega_{k}}\left(\frac{1}{e^{\omega_{k} / T}-1}-\frac{\omega_{k}}{T} \frac{e^{\omega_{k} / T}}{\left(e^{\omega_{k} / T}-1\right)^{2}}\right) \\
& -\frac{i}{8 \pi} \sum_{I} \int \frac{d^{3} k}{(2 \pi)^{3}}\left(\mathcal{T}_{I} \mathcal{T}_{I}^{*^{\prime}}-\mathcal{T}_{I}^{*} \mathcal{T}_{I}^{\prime}\right)(k) \frac{\omega_{k}}{e^{\omega_{k} / T}-1}
\end{aligned}
$$

A comparison between (11) and (44) shows that the real part of the mass shift is in general different from the thermal energy shift, for complex $\mathcal{T}$. This is always the case if the amplitude satisfies unitarity. At low temperatures, however, the pion unitarity cuts are suppressed by powers of $\left(T / f_{\pi}\right)$, and one may argue that the tree calculations may be 
sufficient. Hence that a thermal energy shift becomes a pole mass shift (their difference being an entropy-like term), both of which real. This argument, however, is incorrect given the nearness of the $\Delta$ resonance to the pion-nucleon threshold, and we conclude that the concept of mass shift for the nucleon is process dependent.

\section{Conclusion}

We have analyzed the effects of temperature on the nucleon mass, using the pole-mass definition. To leading order in the density, the mass shift and damping rate were determined exactly to order $1 / f_{\pi}^{2}$ using chiral counting arguments with and without the isobar. For temperature $T$ below the pion mass $m_{\pi}$ where the validity of the virial expansion holds, the role of the $\Delta$-resonance has proved to be the crucial contribution in the mass shift and damping rate of the nucleon. Other effects, like the S-wave scattering length and the one-pion coupling to the nucleon, yield small contributions (of the order of a few percent) compared to this resonance. This result, is in agreement with a virial calculation using the pion-nucleon scattering data [7], and sum rule calculations [20]. A large $N_{c}$ investigation of our results has confirmed the results obtained for the mass shift and damping rate in a heavy baryon chiral perturbation theory $(\mathrm{HB} \chi \mathrm{PT})[8]$. We have shown that unitarity implies that the concept of a mass shift is process dependent.

\section{Acknowledgements}

This work was supported in part by a US DOE grant DE-FG-88ER40388. 


\section{Appendix}

In this appendix, we elaborate on how we introduce the width of the $\Delta$ resonance. We adopt the notations of [14 throughout this appendix. We start with the partial wave decomposition of the direct term in $1 /\left(\nu_{\Delta}-\nu\right)$ in the pole part of the invariant amplitude in 18$)$

$$
A_{l}^{ \pm}(s)=\int_{-1}^{1} d z P_{l}(z) A_{\Delta, d p}^{ \pm}(s, t)
$$

where $z=\cos \theta, \theta$ being the scattering angle in the center of mass frame. $s$ and $t$ are the usual Mandelstam variables and $P_{l}(z)$ the Legendre polynomial of order $l$. We can also write a similar expression for $B_{l}^{ \pm}(s)$. We note that the $t$ dependence in the amplitude is kept in order to correctly project out the waves. This requires the respective change $\alpha_{1} \rightarrow \alpha_{1}+\alpha_{2} t$ and $\beta_{1} \rightarrow \beta_{1}+\beta_{2} t$ in (25) and (26) where $\alpha_{2}=3 q_{\Delta}^{2}-\left(E_{\Delta}+m_{N}\right)^{2}$ and $\beta_{2}=3 / 2$. We obtain

$$
A_{0}^{ \pm}(s)=\left(\begin{array}{c}
2 \\
-1
\end{array}\right) \frac{g_{\Delta}^{2}}{18 m_{N}} 2\left(\alpha_{1}-2 \alpha_{2} q^{2}\right) \frac{1}{\nu_{\Delta}-\nu}
$$

and

$$
A_{1}^{ \pm}(s)=\left(\begin{array}{c}
2 \\
-1
\end{array}\right) \frac{g_{\Delta}^{2}}{18 m_{N}} \frac{2}{3} \alpha_{2} q^{2} \frac{1}{\nu_{\Delta}-\nu}
$$

The respective expressions for $B_{0}^{ \pm}(s)$ and $B_{1}^{ \pm}(s)$ are obtained from $A_{0}^{ \pm}(s)$ and $A_{1}^{ \pm}(s)$ with $\alpha_{1,2} \rightarrow \beta_{1,2}$. All other higher waves vanish. Furthermore, we now write the partial waves of the spin no-flip and spin flip of the full amplitude as follows (the $s$ dependence is understood)

$$
\begin{gathered}
\frac{32 \pi}{3} \sqrt{s} f_{31}^{S}=+\left(E+m_{N}\right)\left(A_{0}^{+}+\left(\sqrt{s}-m_{N}\right) B_{0}^{+}\right) \\
+\quad\left(E-m_{N}\right)\left(-A_{1}^{+}+\left(\sqrt{s}+m_{N}\right) B_{1}^{+}\right) \\
+\quad\left(E-m_{N}\right)\left(-A_{0}^{+}+\left(\sqrt{s}+m_{N}\right) B_{0}^{+}\right) \\
\frac{32 \pi}{3} \sqrt{s} f_{31}^{P}=\quad\left(E+m_{N}\right)\left(A_{1}^{+}+\left(\sqrt{s}-m_{N}\right) B_{1}^{+}\right) \\
\frac{32 \pi}{3} \sqrt{s} f_{33}^{P}=\left(E+m_{N}\right)\left(A_{1}^{+}+\left(\sqrt{s}-m_{N}\right) B_{1}^{+}\right) \\
\frac{32 \pi}{3} \sqrt{s} f_{33}^{D}=\left(E-m_{N}\right)\left(-A_{1}^{+}+\left(\sqrt{s}+m_{N}\right) B_{1}^{+}\right)
\end{gathered}
$$


The width of the isobar is constructed through a Breit-Wigner form for the P33 channel, namely

$$
f_{33}^{P}(q) \rightarrow \frac{f_{33}^{P}(q)}{1-i q \cdot f_{33}^{P}(q)}
$$

With this in hand, we reconstruct the pole part of the amplitude $A_{\Delta, d p}^{+}(\nu)\left(B_{\Delta, d p}^{+}(\nu)\right)$ by inverting the equations for each channel $\alpha$ in $f_{\alpha}$ (49). In turn, we can combine these terms with the crossed terms of the pole part $A_{\Delta, c p}^{+}(\nu)\left(B_{\Delta, c p}^{+}(\nu)\right)$, the non- pole part $A_{\Delta, n p}^{+}(\nu)\left(B_{\Delta, n p}^{+}(\nu)\right)$ and the nucleon contribution $A_{N}^{+}(\nu)\left(B_{N}^{+}(\nu)\right)$ In Figs. $4 \mathrm{a}$ and $4 \mathrm{~b}$, we respectively display in full line the real parts $\operatorname{Re} A^{+}$and $\operatorname{Re} B^{+}$and in Figs. 5a and $5 \mathrm{~b}$ the imaginary parts $\operatorname{Im} A^{+}$and $\operatorname{Im} B^{+}$opposite to the experimental data points [14]. Our procedure is artificial in the sense that it mimics the data in the low energy regime. For instance, the width of the $\Delta$ turns out to be $\Gamma_{\Delta} \sim 90 \mathrm{MeV}$ with the mass $m_{\Delta}=1220$ $\mathrm{MeV}$. This is in contrast with the known values $\Gamma_{\Delta} \sim 114 \mathrm{MeV}$ and $m_{\Delta}=1232 \mathrm{MeV}$. 


\section{Figure captions}

Fig. 1. Nucleon-pole contribution to pion-nucleon scattering.

Fig. 2. $\Delta$-pole contribution to pion-nucleon scattering.

Fig. 3. The various contributions to the nucleon mass shift $\left(a^{+}, \mathrm{N}, \Delta\right)$ are plotted in solid lines. The dotted line exhibits the sum total of these contributions.

Figs. 4a and b. In Figs. 4a and 4b, we respectively display in full line the real parts $\operatorname{Re} A^{+}$and $\operatorname{Re} B^{+}$opposite to the experimental data points (dotted line) [14].

Figs. 5a and b. In Figs. 5a and 5b, we respectively display in full line the imaginary parts $\operatorname{Im} A^{+}$and $\operatorname{Im} B^{+}$opposite to the experimental data points (dotted line) [14].

Fig. 6. The nucleon mass shift is plotted (full line) opposite to the one obtained from the experimental data points (dotted line) [14].

Fig. 7. The nucleon damping rate is plotted (full line) opposite to the one obtained from the experimental data points (dotted line) [14.

Fig. 8. The pole (p) and non-pole (np) contributions to the mass shift is displayed. The short-dashed line represents the sum of the pole and non-pole contribution and the long-dashed line the mass shift from chiral power counting of Fig. 6.

Fig. 9. The damping rate (40) in the large $N_{c}$ limit is displayed in full line opposite the result obtained from chiral power counting (long-dashed line). 


\section{References}

[1] R. D. Pisarski, Phys. Lett. B100(1982)155; T. Hatsuda and T. Kunihiro, Phys. Rev. Lett.55(1985)158; V. Bernard, U. -G. Meissner and I. Zahed, Phys. Rev. Lett.59(1987)966; G. Ripka and M. Jaminon, Ann. of Phys. (N.Y.)218(1992)51. L. Ya. Glozman and D. O. Riska, The Baryon spectrum and Chiral Dynamics, preprint HU-TFT-94-47, [LANL] hep-ph 9411279 (1994); Systematics of the Light and Strange Baryons and the Symmetries of QCD, preprint HU-TFT-94-48,[LANL hep-ph 9412231 (1994) ; The spectrum of the Nucleons and the Strange Hyperons and Chiral Dynamics, preprint DOE/ER/40561-187-INT95-16-01 [LANL hep-ph 9505422], Phys. Rep. in print.

[2] A. I. Bochkarev and M. E. Shaposhnikov, Nucl. Phys. B268(1986)220; H. G. Dosh and S. Narison, Phys. Lett. B203(1988)155; R. J. Furnstahl, T. Hatsuda and S. H. Lee, Phys. Rev. D42(1990)1744; C. Adami, T. Hatsuda and I. Zahed, Phys. Rev. D43(1991)921; T. Hatsuda and S. H. Lee, Phys. Rev. C46(1992)34; T. Hatsuda, Y. Koike and S. H. Lee, Nucl. Phys. B394(1993)221; C. A. Dominguez, M. Loewe and J. C. Rojas, Z. Phys. C59(1993)63 C. A. Dominguez and M. Loewe, hep-ph/9406213.

[3] G. Q. Li, C. M. Ko and G. E. Brown, 'Dilepton emission', (in preparation).

[4] G. Apakichiev et al. Phys. Rev. Lett. 75(1995)1272.

[5] M. Masera, Nucl. Phys. A590(1995)93C.

[6] H. Yamagishi and I. Zahed, preprint SUNY-NTG-94-57, Ann. of Phys. in print.

[7] H. Leutwyler and A. V. Smilga Nucl. Phys. B342(1990)302.

[8] P. F. Bedaque, preprint MIT-CTP-25XX [LANL hep-th/9511365].

[9] R. Koch, Nucl. Phys. A448(1986)707.

[10] S. Weinberg, 'Lectures on Elementary Particles and Quantum Field Theory', Brandeis Summer Institute 1970, S Deser, M. Grisaru and H. Pendleton, M.I.T. Press 1970.

[11] J. Steele, H. Yamagishi and I. Zahed, preprint SUNY-NTG-95-11.

[12] D. Lurie, 'Particles and Fields', Interscience Publishers ( J. Wyley and Sons)1968.

[13] M. Soldate, Fermilab-PUB86/59-T.

[14] G. Höhler, p. 102, 500, 561 in Landolt-Börnstein, vol. 9b2. ed. H Schopper (SpringerVerlag, 1983); G. Höhler, H. P. Jakob and R. Strauss, Nucl. Phys. B39(1972)237.

[15] E. Oset, h. Toki and W. Weise, Phys. Rep. 83(1982)281.

[16] J. Gasser, H. Leutwyler and M. E. Sainio, Phys. Lett. B253(1991)260. 
[17] M. G. Olsson, L. Turner and E. T. Osypowski, Phys. Rev. D11(1973)3444; M. G. Olsson and E. T. Osypowski, Nucl. Phys. B101(1975)136.

[18] M. Kacir and I. Zahed, preprint SUNY-NTG-95-54.

[19] L. D. Landau and E. Lifchitz, Statistical Physics, Pergament Press (1980).

[20] C. Adami and I. Zahed, Phys. Rev. D45(1992)4312. 


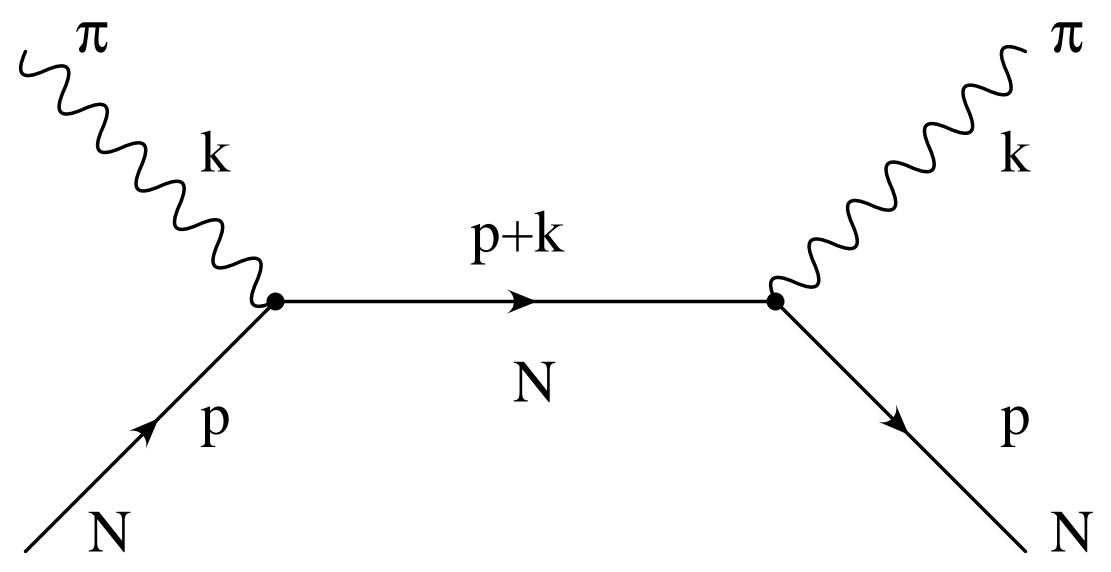

(a)

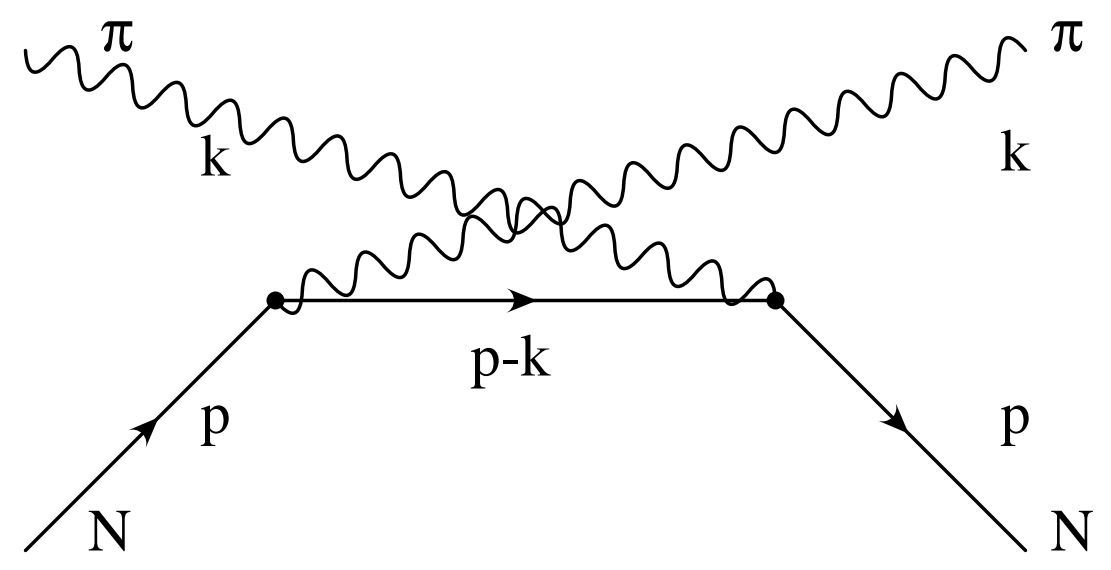

(b)

Fig.1 


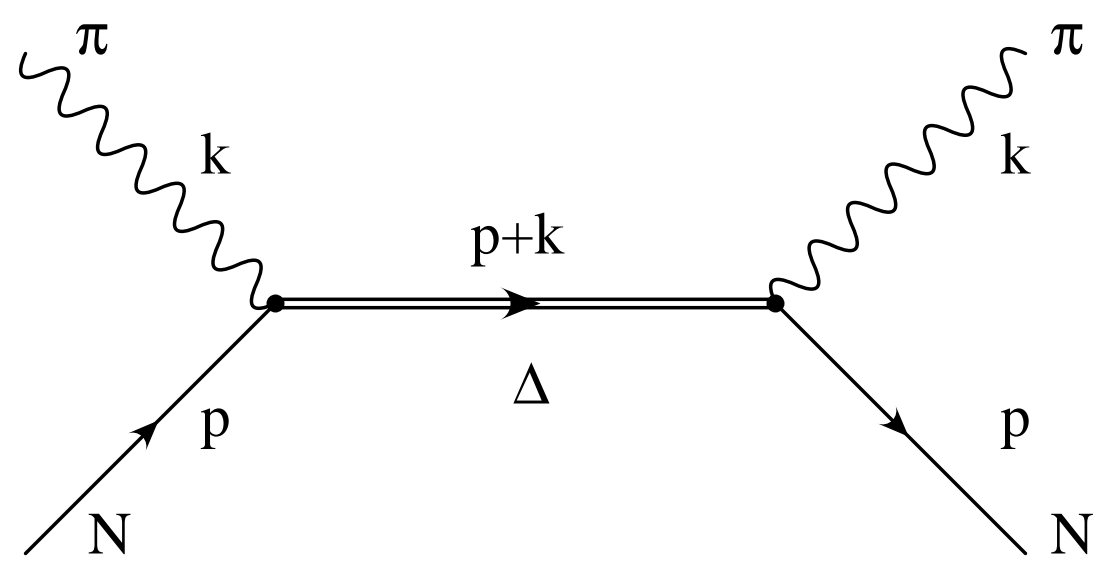

(a)

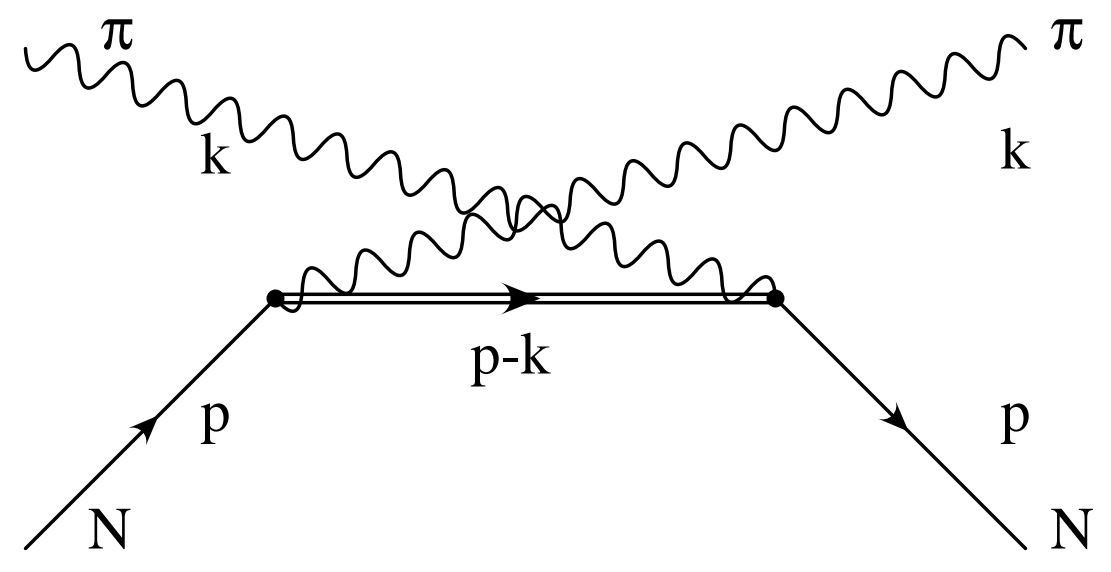

(b)

Fig.2 


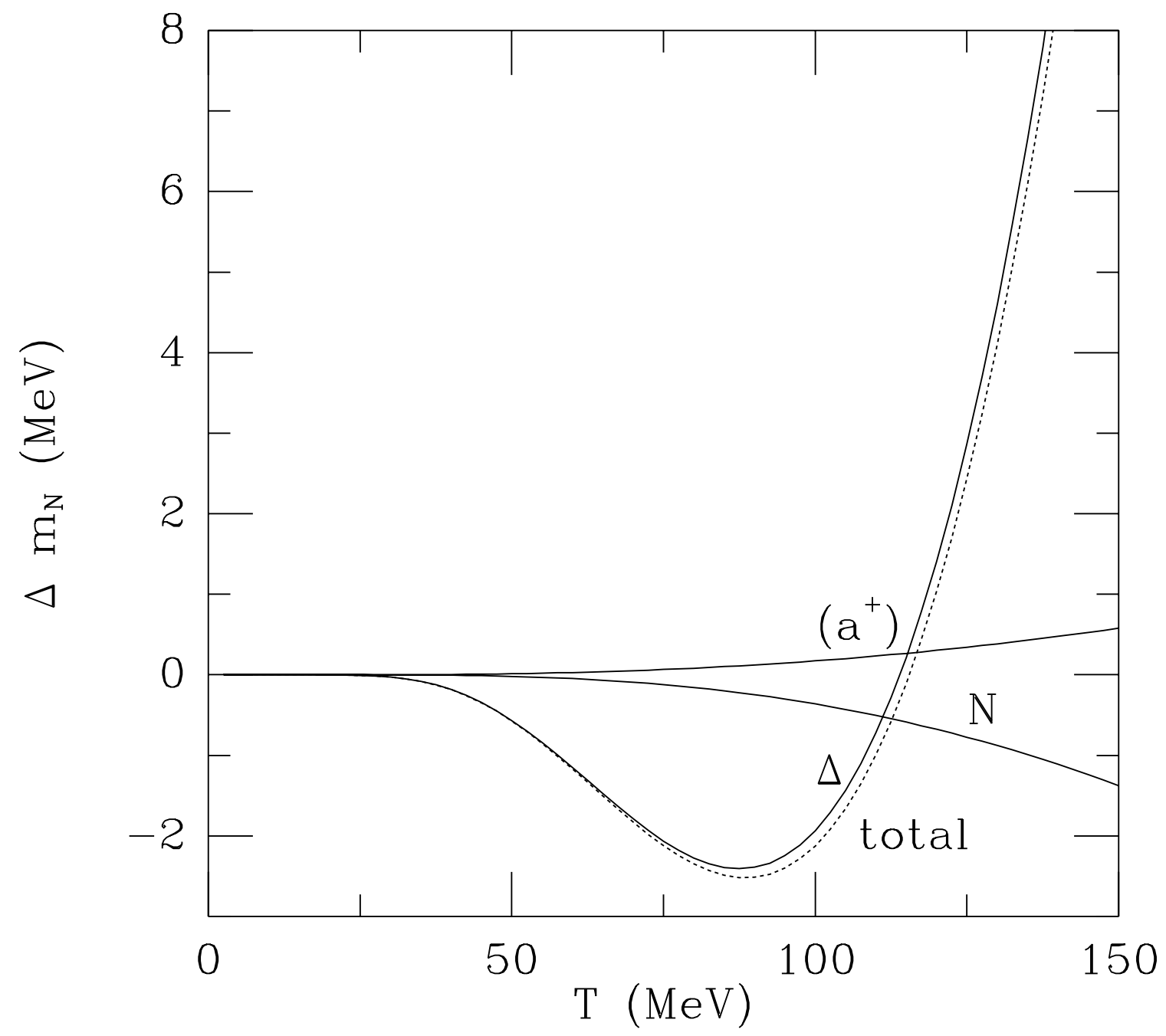

Fig. 3 


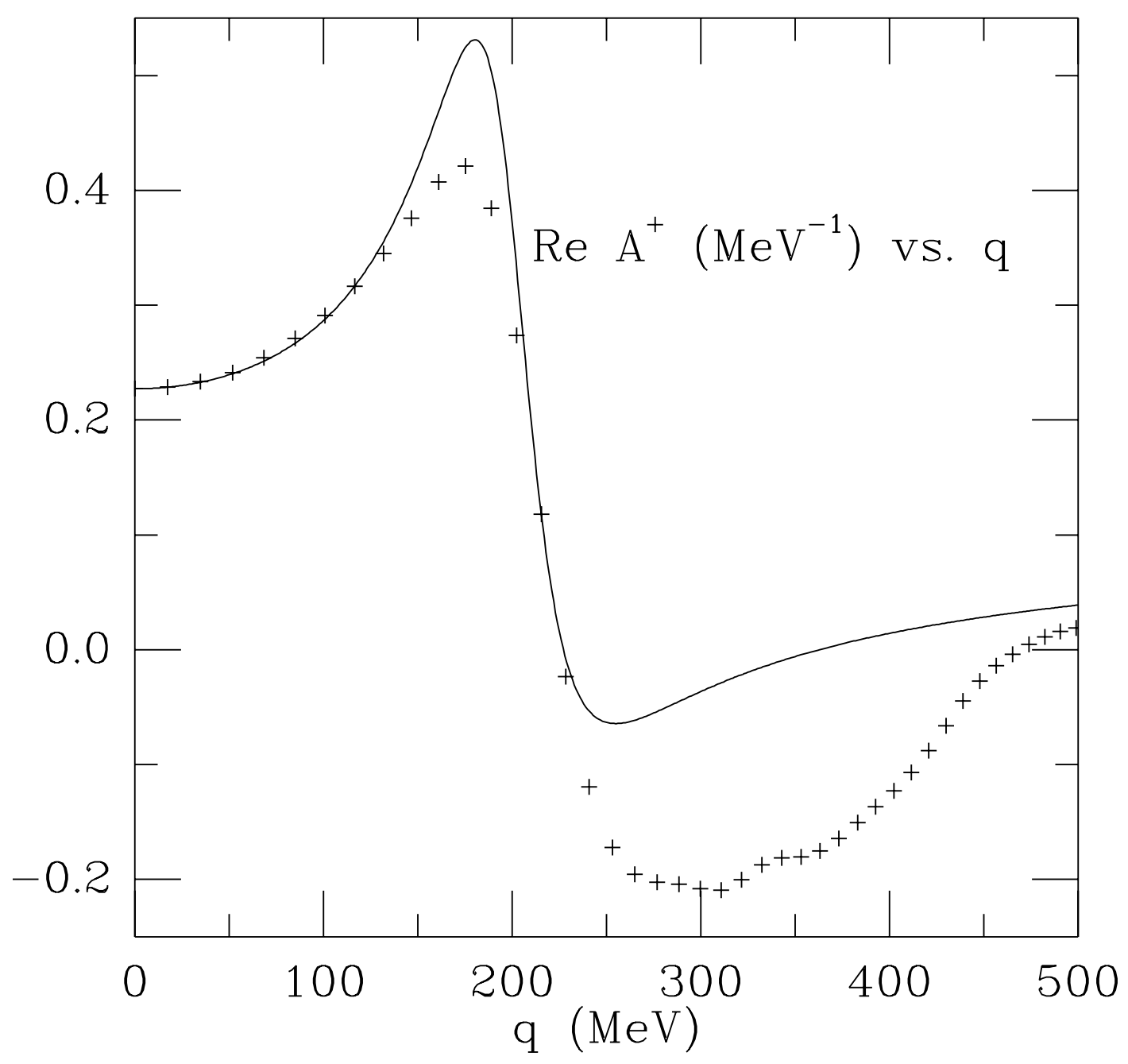

Fig. $4 a$ 


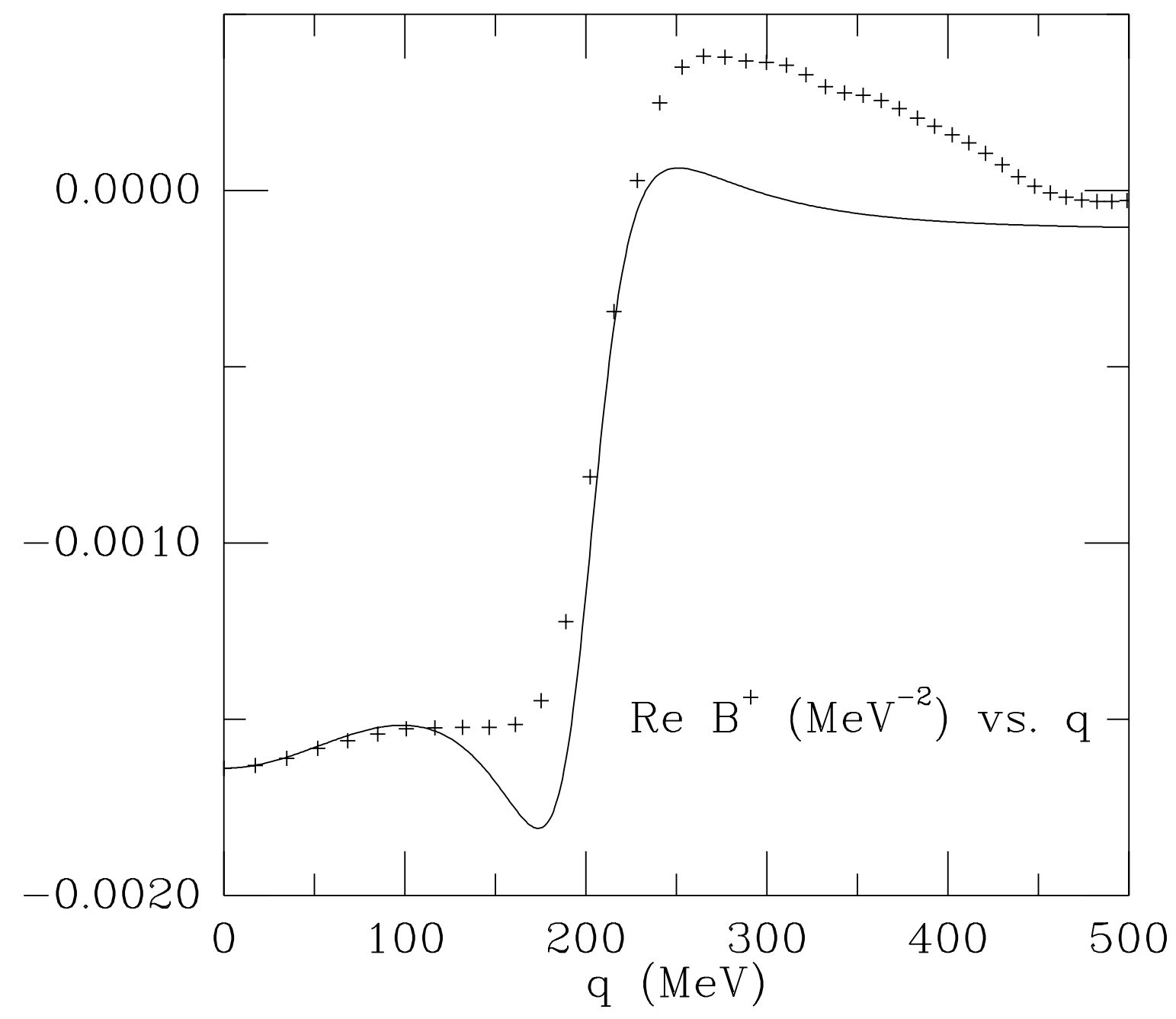

Fig. 4b 


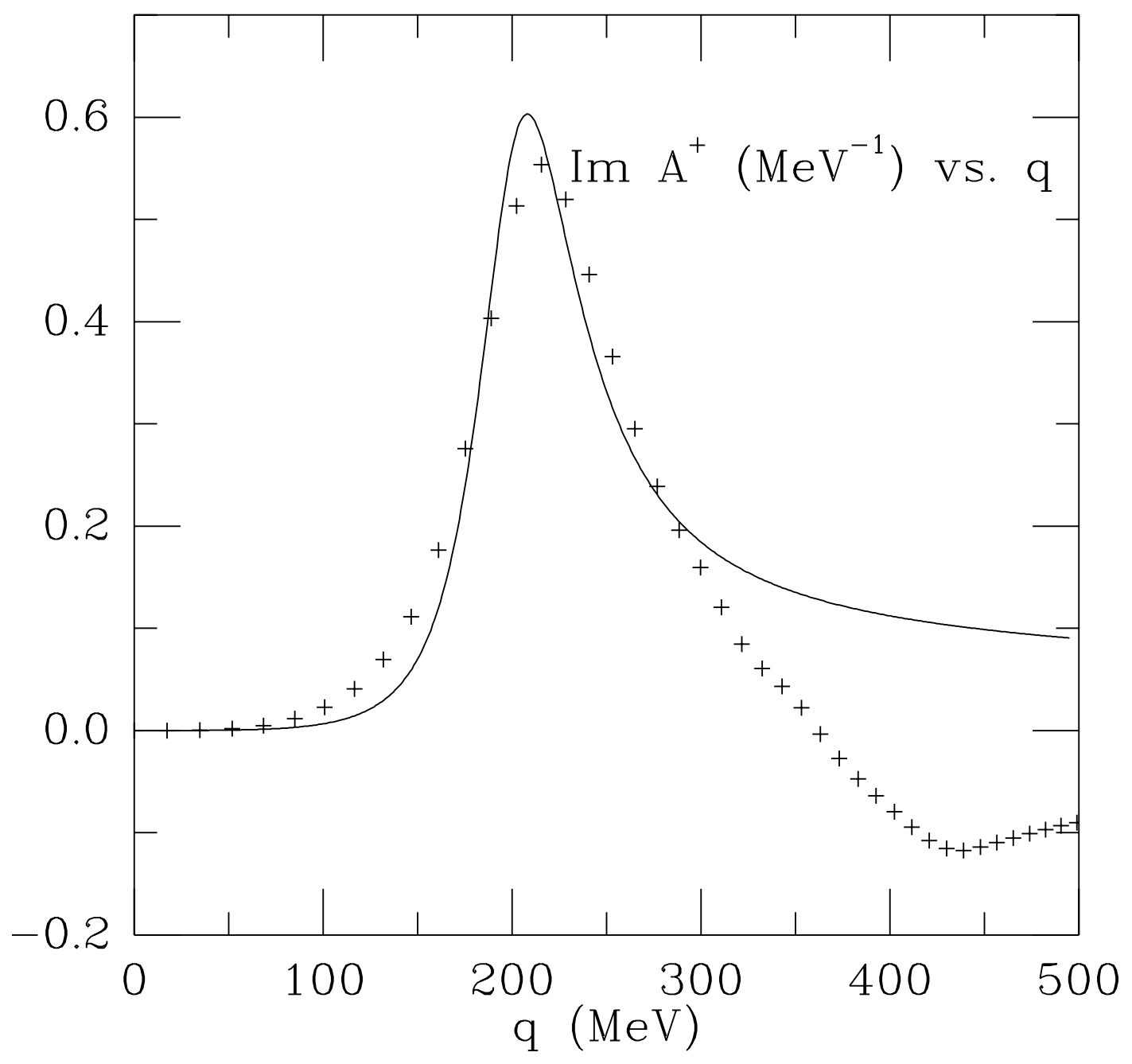

Fig. 5a 


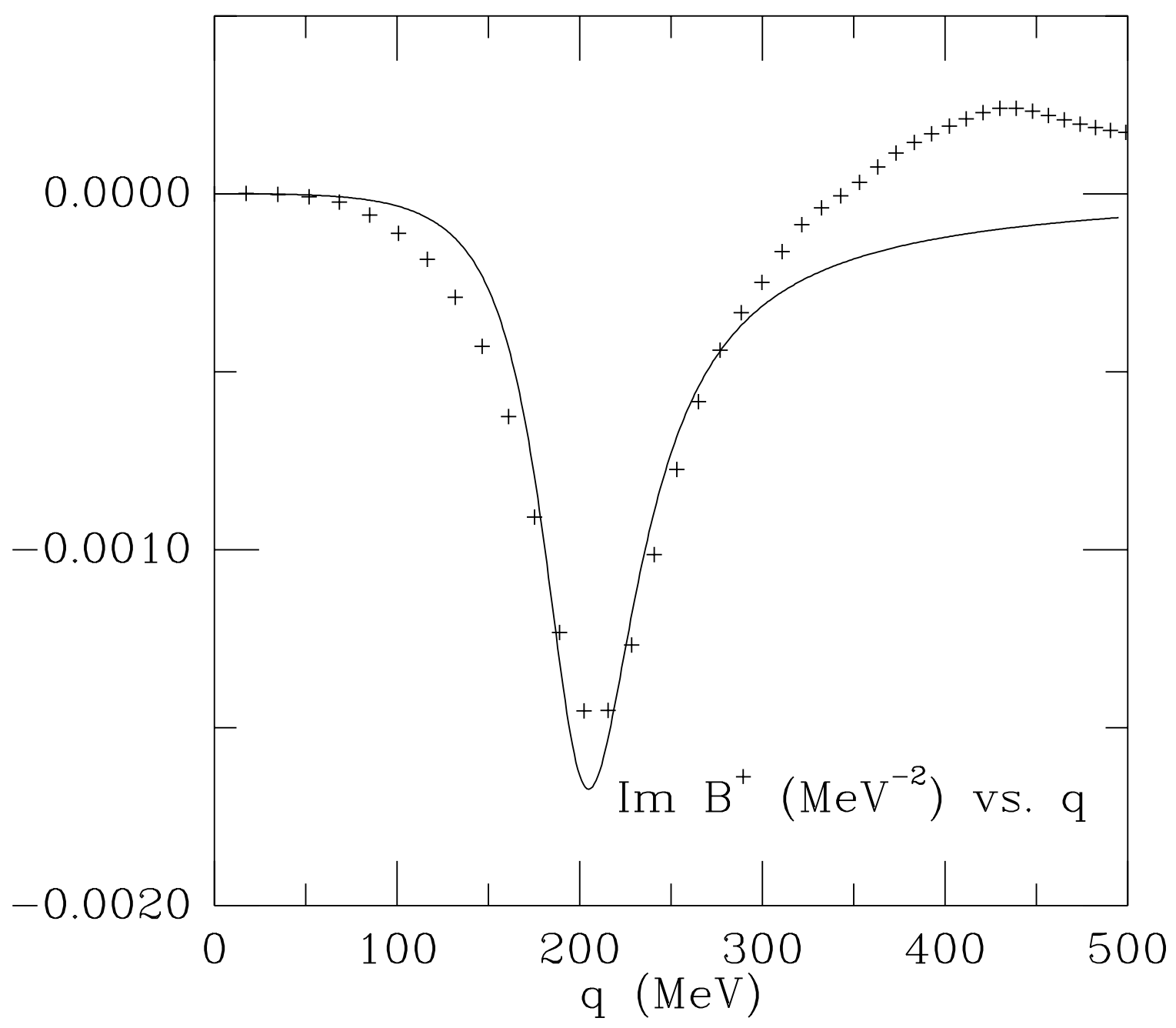

Fig. 5b 


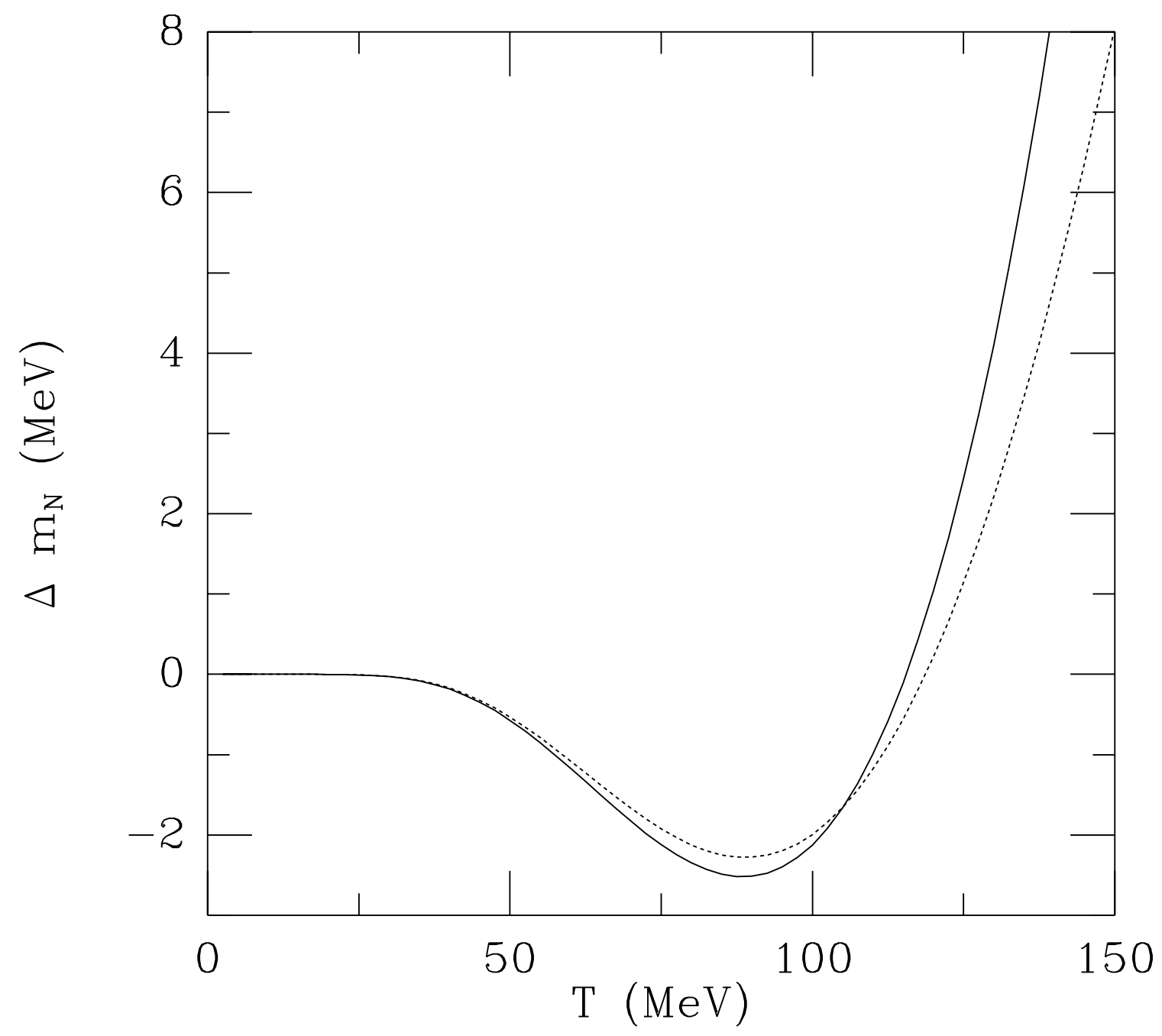

Fig. 6 


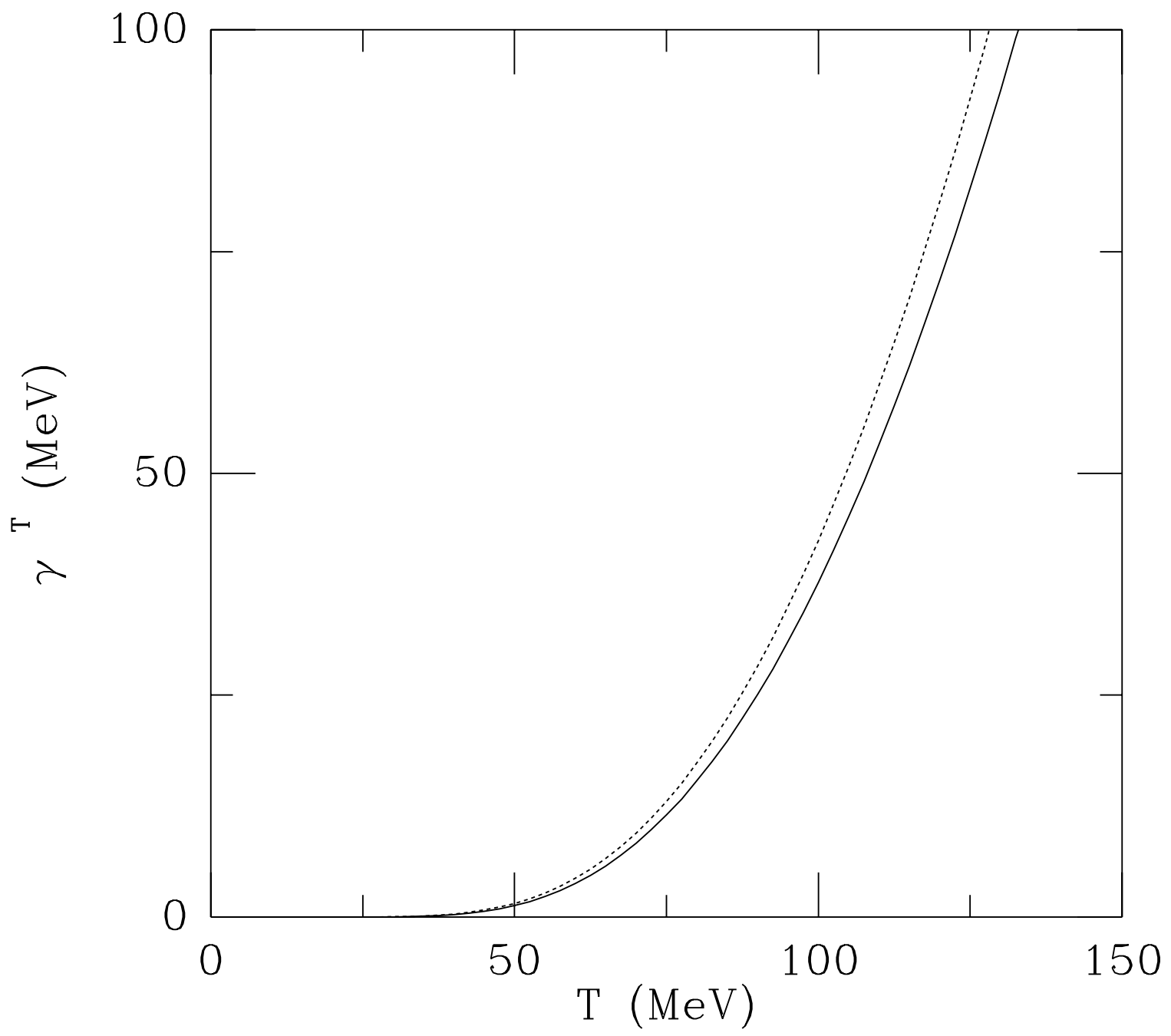

Fig. 7 


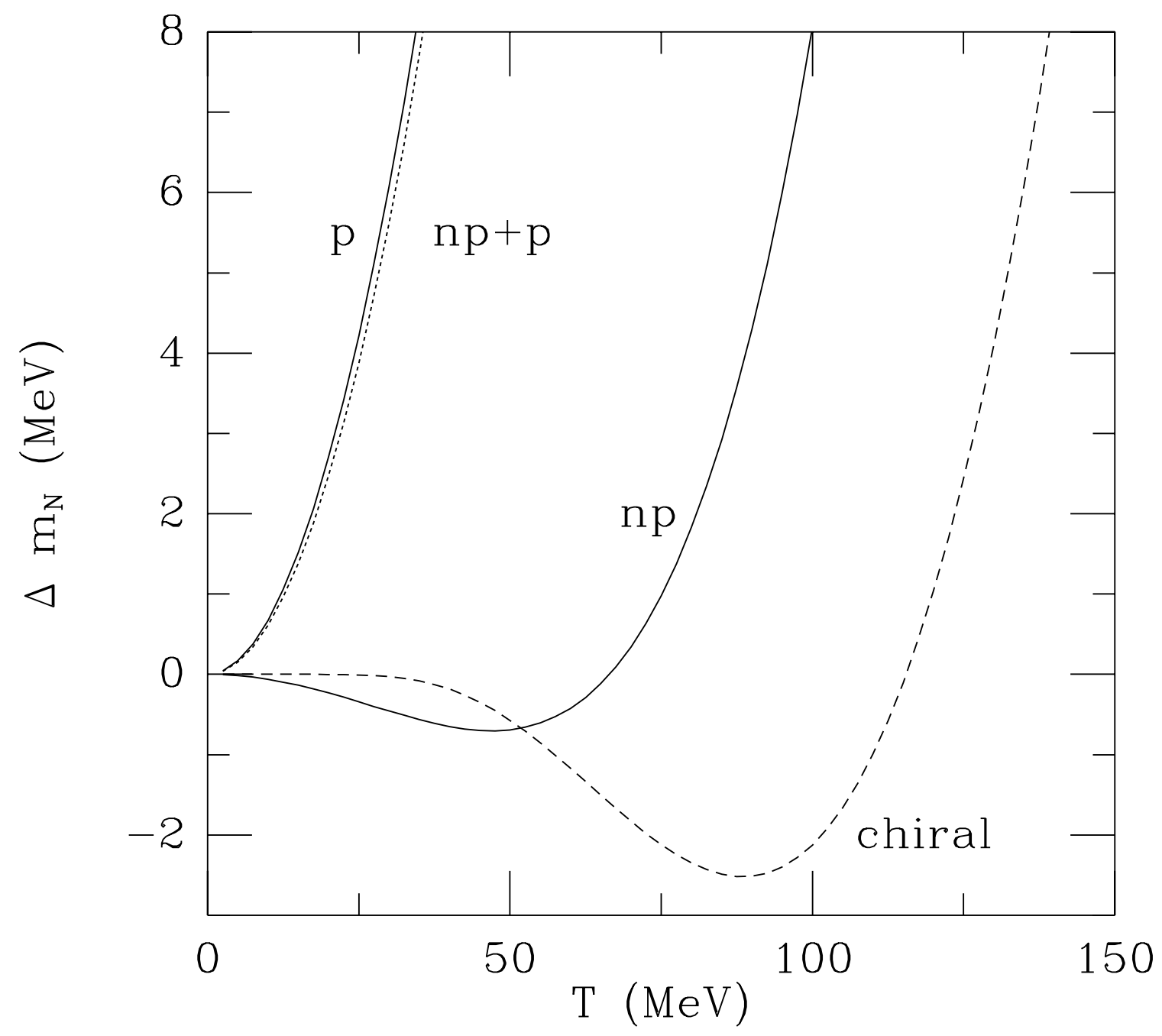

Fig. 8 


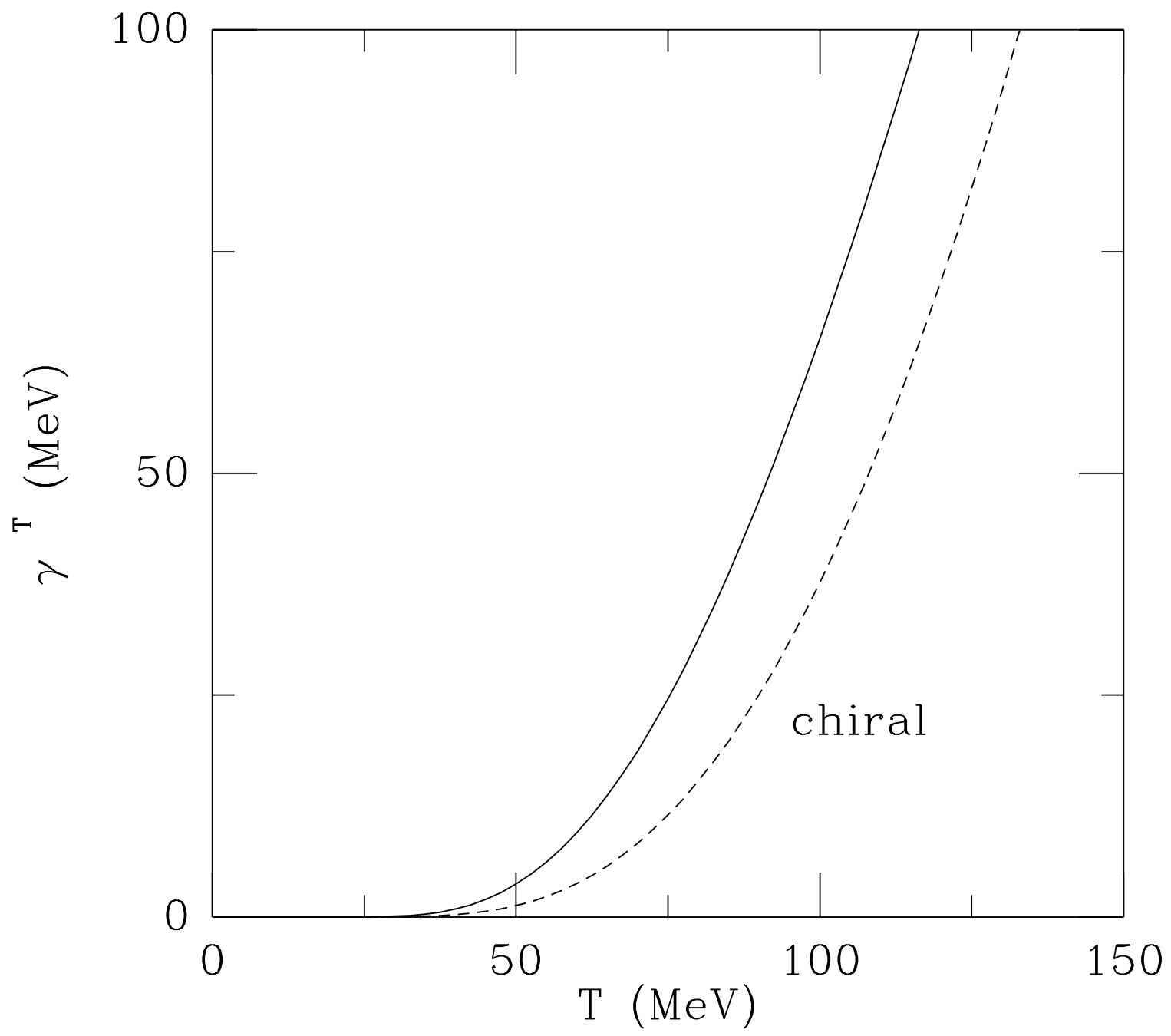

Fig. 9 